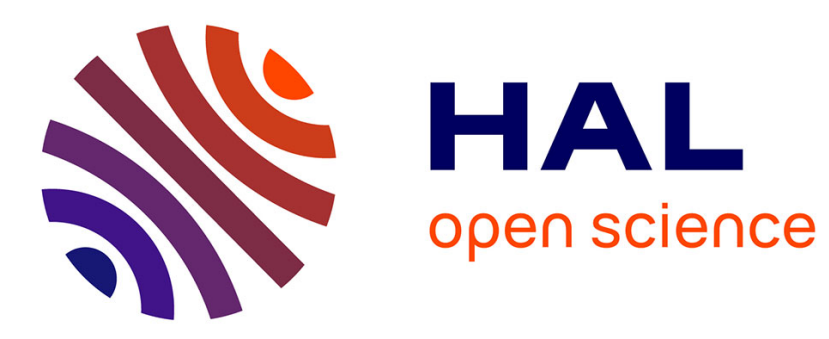

\title{
Mobility management in Multihops Wireless Access Networks
}

Fabrice Theoleyre, Fabrice Valois

\section{To cite this version:}

Fabrice Theoleyre, Fabrice Valois. Mobility management in Multihops Wireless Access Networks. PWC 2005 - International Conference on Personal Wireless Communications, Aug 2005, Colmar, France. 10.1142/9781860947315_0016. inria-00406111

\section{HAL Id: inria-00406111 https://hal.inria.fr/inria-00406111}

Submitted on 12 May 2020

HAL is a multi-disciplinary open access archive for the deposit and dissemination of scientific research documents, whether they are published or not. The documents may come from teaching and research institutions in France or abroad, or from public or private research centers.
L'archive ouverte pluridisciplinaire HAL, est destinée au dépôt et à la diffusion de documents scientifiques de niveau recherche, publiés ou non, émanant des établissements d'enseignement et de recherche français ou étrangers, des laboratoires publics ou privés. 


\title{
MOBILITY MANAGEMENT IN MULTIHOPS WIRELESS ACCESS NETWORKS
}

\author{
FABRICE THEOLEYRE AND FABRICE VALOIS \\ CITI - INRIA ARES, INSA Lyon \\ 21, Avenue Jean Capelle, 69621 Villeurbanne Cedex, France \\ Email: \{fabrice.theoleyre, fabrice.valois\}@insa-lyon.fr \\ Tel: (+33) 472436327 and (+33) 472436418 Fax: (+33) 4 724362 27
}

\begin{abstract}
Ad hoc networks are spontaneous networks of mobile nodes which use wireless communications. The nodes must collaborate to route and forward data packets from a source to a destination. We consider multihops wireless access networks which are ad hoc networks connected to the Internet via Access Points (AP). A terminal can send and receive data packets to and from the Internet. To achieve this goal, we propose here a micro-mobility management solution. The solution uses a virtual backbone to disseminate information and to limit the overhead. The proposition mixes the reactive and the proactive approaches to propose a trade-off between the delay and the overhead. In upload, each node has a default proactive route toward the AP introducing no latency. In download, the AP initiates a reactive localization to find a route to the destination inside the ad hoc area, reducing the overhead. A solution of paging with several AP and a solution of power-energy saving are also proposed.
\end{abstract}

\section{Introduction}

Mobile Ad hoc networks (MANET) could be defined as spontaneous networks: a collection of terminals organizes itself to exchange packets with each other via wireless communications. A source is not always a neighbor of the destination, so a route must be provided. Moreover, neither wired nor wireless router exist to manage the network. Thus, some terminals must collaborate to forward the data packets from the source to the destination. Ad hoc networks do not distinguish the routers and the clients: a terminal plays both roles. In a MANET, all terminals are independent and can move freely. MANET could be interconnected to the Internet via special gateways: the Access Points (AP). Such networks are often called Hybrid Networks or Multihops Wireless Access Networks.

Routing is one of the major issues in the MANET: a packet must be forwarded from a source to a destination without loss and with reduced 
delay and overhead. Many protocols were proposed and can be classified in 2 main categories: the reactive protocols discover routes on-demand, flooding the network to find a route, whereas in proactive protocols, each node floods the network to create proactively routes toward it. Because of the particular constraints of MANET, we think that a self-organization is required. Some nodes must be chosen to manage the network, helping weakest nodes. These managers will stabilize the logical network view and will allow to reduce the control traffic. A self-organization ${ }^{6}$ was already proposed: strongest nodes form a virtual backbone, acting as routers and managers. This backbone can optimize floodings and reduce the control traffic for other nodes. We propose to use this virtual backbone to provide a new solution for mobility management. The data packets are forwarded proactively from the source toward the AP, constituting a default route to Internet. Inversely, routes from the AP toward a node could be discovered reactively. A solution of paging and power-energy saving are also proposed.

Next, we expose related work about routing in hybrid networks. Section 3 presents briefly the virtual backbone and the routing solution. Paging and power-energy saving solutions are also described. Section 4 presents some simulation results, before the conclusion and perspectives.

\section{Related Work}

MIPMANET ${ }^{3}$ proposes to integrate a reactive routing protocol (AODV) and Mobile IP. AODV is used for internal communications whereas external communications use Mobile IP. The Access Point acts as Mobile IP gateway (Foreign Agent): it must periodically flood Agent advertisements in the network, giving some Mobile IP parameters. Floodings presents many problems in MANET ${ }^{4}$ : redundancy of transmissions exist and collisions occur creating a lack of reliability. The advertisements could be discovered reactively by clients ${ }^{5}$, but inducing a delay. A proactive and reactive combination is also possible, but the trade-off is complex to set. Moreover, a node must flood several times the network before deciding that the destination is outside the MANET area.

To the best of our knowledge, only MEWLANA ${ }^{1}$ proposes a solution optimized only for hybrid networks. The authors present 2 approaches. MEWLANA-TD allows both internal and external communications and is inspired from MIPMANET, but using a proactive routing protocol (DSDV). Each node knows instantaneously a route toward each node in the MANET area. However, the overhead for Agent Advertisements re- 
mains unchanged. Moreover, the ratio of internal communications must be sufficient to justify the important control traffic amount required by DSDV. The second approach, MEWLANA-RD, is specifically designed for hybrid networks. The AP sends periodically Agent Advertisements. Each node registers the forwarder as the default route to the AP and forward the Agent Advertisement. In parallel, each node answers in sending a Registration to the AP, on the new default route. However, the periodical Agent Advertisements and Registrations overload the radio medium, and many collisions occur, inducing many packet losses. Moreover, the systematic periodical reconstruction of the tree is suboptimal.

\section{Proposition}

Our solution mainly focus on the problems of both routing and mobility management in hybrid networks. The AP being the gateway to the Internet, it may constitute the default route, routing all the traffic, and acting as a Mobile IP Foreign Agent. If the AP has packets to deliver to one of its nodes with no associated route, one is discovered reactively.

We use a virtual backbone ${ }^{6}$. The backbone nodes, the dominators, are selected according to a stability weight representing their aptitude to act as network managers. Some nodes are elected as dominators to form a connected structure where each normal node (or dominatee) is at most $k$ hops far from its dominator. The backbone constitutes a tree of dominators where the leaves are the dominatees. Each dominator maintains the identity of its parent (except the AP) and the identity of the dominators for which it is a parent: they constitute its children. MANet are volatile environments. Hence, we have proposed a maintenance protocol to maintain the efficiency of the virtual backbone. To maintain the backbone connectivity, AP-hello are periodically sent by the AP, but forwarded only by dominators, limiting the overhead. Procedures for backbone reconnections are also proposed.

\subsection{Mobility Management}

\subsubsection{Upload}

The AP can represent a suited default router. When a node wants to send a packet, it delivers it to the AP. Then, the AP acts as a proxy to find a route in the Internet, to do Network Address Translation if required,... The backbone is a tree rooted at the AP where a parent represents the next hop through the backbone to reach the AP. Each nodes maintains proactively 
the identity of its k-neighbors, with classical hello packets. A dominatee knows the identity of its dominator, its distance, and a next hop toward it. This next hop can appears to be the default route. A dominator maintains the identity of its parent in the tree, from which it receives the ap hellos. This parent represents its default route. We assume that communications will mainly be initiated by the nodes creating an efficient proactive feature: the route knowledge requires no latency and no additional overhead. An inverse route can be learned gratuitously, as described further.

\subsubsection{Download}

When a data packet is received from the node $N$, the node registers $N$ as the next hop toward the source before forwarding the packet. Hence, an inverse route, i.e. in download, can be gratuitously learned when a node sends a data packet. Each data packet refreshes this proactive route, in triggering the associated timer in its cache.

However, if the node did not send a data packet, or if the timer of the route expired, the AP must implement a localization process. Because we assume this case seldom, we propose a reactive solution. When the AP receives a packet, and no route to the destination $D$ is known, it buffers the data packet, the memory of the AP being supposed to be important. Then, the AP sends in multicast to its children in the backbone a Route Request. Each dominator $N$ which receives this packet forwards the request if $D$ is unknown. Else, $N$ acts as proxy and sends a Route Reply to the AP, the source being seen as $D$. The Route Request is forwarded along the backbone until $D$ is found. If $D$ is a dominator, one of its dominator neighbor or in the worst case the node itself will send a Route Reply. In the same way, if $D$ is a dominatee, its dominator is at most $\mathrm{k}$ hops far. Hence, $D$ is in the neighborhood table of its dominator: a Route Reply will be generated. To limit the impact of the backbone disconnections, any dominator is allowed to act as proxy for Route requests. This limits the Route Request failure, at the cost of a negligible overhead. The Route Reply is sent on the default route, to the AP. A route is hop by hop created in the cache of each intermediary dominator, like with data packets. A distributive route cache is created, a timer being associated to this route. To adapt the solution to topology changes, a dominator which loses one of its children send a Route Delete. This packet, forwarded to the AP, deletes all outdated routing entries. 


\subsubsection{Ad Hoc Routing}

As an extension, ad hoc connectivity can be proposed. A source $S$ sends a data packet to the destination $D$ via its default route toward the AP. If a node receiving it knows a route to the destination, it sends directly the packet along this route. Else, the AP will finally receive the data packet. The AP will know if $D$ is in the Internet or in the ad hoc area (with the address prefix, its own paging cache...). If $D$ is in its covering area, it adds the data packet to the data buffer, and sends a Route Request. When the Route Reply arrives, the AP sends the buffered packets. Other data packets to $D$ will be forwarded until it reached the first common ancestor of $D$ and the source in the backbone. The route length and the delay are in conclusion not optimal.

\subsection{Paging and Power-energy saving Schemes}

Paging is used in cellular networks to limit the overhead of registrations. The node registers itself less frequently in its paging zone than in its AP. Paging consists in finding the AP serving a destination. A Paging Master (PM) is connected to all the AP constituting the paging zone. The Paging Master adds the mobile in its Membership Cache with a long timeout. When a packet arrives, the PM verifies that the destination is present in its Membership Cache. Then, it searches an associated entry in the Paging Routing Cache associating an AP to a node. If an entry is found, the data packet is directly sent to the corresponding AP. Else, the PM buffers the data packet and sends a Paging Request to all AP of its paging zone. These AP will send a Route Request. When an AP receives a Route Reply, it sends a Paging Ack to the PM. The PM adds an entry in its Paging Routing Cache with a short timeout and sends the buffered packet.

Terminals have limited energy reserves. However, turn off its radio is the only way to economize its energy ${ }^{2}$. Such a node does not participate in the network life, it sleeps. The backbone is particularly suited for such a feature. Elections are based on a weight depending on the energy reserve. Thus, a node with a too low energy reserve will not be elected as backbone member. Moreover, dominatees have a role of clients, they can spare their energy. The degree must be sufficient so that eventual backbone reconnections could be forwarded. Thus, a dominatee is allowed to sleep if the number of not-sleeping 1-neighbors is superior or equal to 6 . Finally, a dominatee is allowed to sleep according to the probability $1 / n, n$ being the number of 1-neighbors with a lower weight. 


\section{Performance Evaluation}

We simulate our solution wit OPNET Modeler 8.1, with the WIFI standard model (300m radio range), and the random waypoint mobility model. The default parameters are a speed of $5 \mathrm{~m} \cdot \mathrm{s}^{-1}, 40$ nodes and a degree of 9 . Data flows of 8 data packets interspaced by $0.25 \mathrm{~s}$ are sent according to the exponential distribution with a mean value of $2 \mathrm{~s}$. Data flows are sent in the same way from the AP to one random destination, and from a random source to the AP. The maximum distance from one node to the backbone is 2 hops. To evaluate the solution, we investigate the behavior according to the mobility, the load, the number of nodes, with the paging and the power-energy saving activated or not. We compare MEWLANA-RD and the proposed solution, labeled $c d c l$.

Figure 1. Impact of the mobility on performances

Our solution is robust according to the mobility: the delay increases slowly, even with a very high speed of $30 \mathrm{~m} . \mathrm{s}^{-1}$. The delay of MEWLANA$\mathrm{RD}$ follows the same tendencies. Our solution mixing the reactive and the proactive approaches doesn't suffer from the delay compared to the fullproactive approach. The delivery ratio is almost symmetrical in upload and in download. Oppositely, the delivery ratio in download is in MEWLANARD 10\% lower than in upload. MEWLANA-RD reconstructing periodically the whole backbone, collisions occur. Finally, the delivery ratio in both directions is higher for cdcl thant for MEWLANA-RD.

Figure 2(a) presents the horizontal scalability of both solutions, i.e. the performances when the number of nodes increases. The delivery ratio of cdcl is higher than that of MEWLANA-RD. Moreover, the symmetrical property of the delivery ratio remains identical for cdcl. Both solutions are simulated with a constant degree when the number of nodes increases. Hence, the average route length growth when the number of nodes increases, increasing the delay too. However, this increasing remains acceptable. 
(a) impact of the number of nodes

(b) impact of load

Figure 2. Scalability

Figure 2(b) presents the performances of both solutions according to the load of the network, i.e. the number of simultaneous communications. We can observe that both approaches are very scalable according to the load for the delivery ratio, although MEWLANA-RD keeps on presenting more packet losses. The delay increases lightly when the load becomes very important, when every node is in communication. However, such an augmentation remains below $50 \mathrm{~ms}$. Finally, we can note that the overhead of MEWLANA-RD (1.4 packet per second) is higher than for cdcl (0.8 pps).

We implement our solution of paging, creating a Paging Master. We place $2 \mathrm{AP}$ on the surface, on the top left and top right corners. This network can deal with a more important load, the data packets being on average distributed among the AP, which is the requested property (fig.3(a)). However, the delivery ratio suffers more from the mobility. The delay of convergence of all routing caches and paging caches could be important, some data packets are dropped.
(a) Paging
(b) Power-energy saving

Figure 3. Impact of the Paging and the Power-energy-saving solutions

The power-energy solution was simulated. Third of the nodes don't generate or receive data packets, they can sleep if they are dominatees. 
This power-energy saving solution has almost no impact on the delay, but the delivery ratio decreases slightly. If a collision occurs for an hello, the neighbors can choose as next hop a sleeping node, creating data packets losses. A non-communicating node is sleeping on average $25 \%$ of the time.

\section{Conclusion}

We propose here a routing and localization solution optimized for hybrid networks. This solution uses a virtual backbone to structure routing caches. In upload, information to maintain the backbone is used to find a route to the AP, constituting a gratuitous default route. In download, the AP implements a localization procedure to discovers a route reactively. To minimize the frequency of the localization process, an inverse route is created on the fly when a node sends a Data Packet toward Internet. The solution presents a very high delivery ratio, and a limited delay although a reactive solution is used besides the proactive part. A solution of paging and power-energy saving are also proposed, taking into account the natural heterogeneity of the hybrid network. The backbone hides many topology changes, offering a stable view of the topology. Next step of this study will be the implementation of new functions like the handover with the choice of the optimal AP, the load balancing among the AP according to the number of nodes and a multicast routing solution.

\section{References}

1. M. Ergen and A. Puri. Mewlana-mobile ip enriched wireless local area network architecture. In Vehicular Technology Conference (VTC), volume 4, pages 2449-2453. IEEE, 2002.

2. L. M. Feeney and M. Nilsson. Investigating the energy consumption of a wireless network interface in an ad hoc networking environment. In INFOCOM, volume 3, pages 1548-1557. IEEE, 2001.

3. U. Jonsson, F. Alriksson, T. Larsson, P. Johansson, and G. Q. Maguire. Mipmanet-mobile ip for mobile ad hoc networks. In Workshop on Mobile and Ad Hoc Networking and Computing (MobiHOC), pages 75-85. ACM, 2000.

4. Sze-Yao Ni, Yu-Chee Tseng, Yuh-Shyan Chen, and Jang-Ping Sheu. The broadcast storm problem in a mobile ad hoc network. In Mobicom. ACM, 1999.

5. Y. Sun, E.M. Belding-Royer, and C.E. Perkins. Internet connectivity for ad hoc mobile networks. International Journal of Wireless Information Networks, 9:75-88, 2002.

6. F. Theoleyre and F. Valois. A virtual structure for hybrid networks. In Wireless Communications and Networking Conference (WCNC), volume 2, pages 10401045. IEEE, 2004. 